\title{
Gliquidone Alleviates Diabetic Nephropathy by Inhibiting Notch/Snail Signaling Pathway
}

\author{
Hengyu Tian Junbo Yang Zhuochao Xie Jialin Liu \\ Department of Hepatobiliary Surgery, Shenzhen Key Laboratory of Hepatobiliary Disease, Shenzhen \\ Third People's Hospital, China
}

\section{Key Words}

Diabetic nephropathy $\cdot$ Gliquidone $\cdot$ Renal tubular epithelium $•$ EMT $\cdot$ Renal interstitial fibrosis - Notch/Snail1 signaling pathway

\begin{abstract}
Background/Aims: Diabetic nephropathy is a common complication of diabetes. This study explored the renal protective effect and possible mechanism of gliquidone in mice with diabetic nephropathy. Methods: Animal model of diabetic nephropathy was established in KKAy mice. The renal protective effect of gliquidone was studied by evaluating the kidney function through measures of urinary protein, blood urea nitrogen (BUN), serum creatinine (Scr) and serum triglyceride (TG) that were performed using an automatic biochemical analyzer. The levels of oxidative stress indicators, such as nitric oxide (NO), superoxide dismutase (SOD) and malondialdehyde (MDA), were evaluated in renal tissue homogenates using the automatic biochemical analyzer. The inhibitory effect of gliquidone on renal interstitial fibrosis and its association with Notch / Snail1 signaling pathway in diabetic nephropathy was investigated using molecular biological techniques. Results: It was found that low-, medium- and highdose gliquidone improved the mice's general health condition, such as mental status, fur condition, eating, and drinking. Gliquidone reduced the body weight and the kidney weight / body weight ratio of mice. Gliquidone improved the kidney function, indicated by reductions in urinary protein, blood urea nitrogen, and serum creatinine and triglyceride. Gliquidone treatment increased levels of nitric oxide and superoxide dismutase, but decreased level of malondialdehyde. The expression of Jagged1/Notch1/hes1/Snail1/ $\alpha$-SMA decreased, while the expression of E-cadherin increased in gliquidone-treated kidneys. High dose gliquidone showed the best effect, one that was similar to that of the positive control drug irbesartan. Conclusion: Taken together, our results suggested that gliquidone can ameliorate the diabetic symptoms of diabetic nephropathy through inhibiting Notch / Snail1 signaling pathway, improving anti -oxidative response and delaying renal interstitial fibrosis. The efficacy of gliquidone is dose-dependent.




\section{Cellular Physiology Cell Physiol Biochem 2018;51:2085-2097

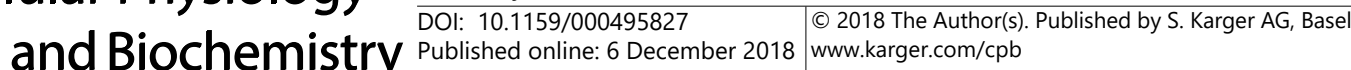 \\ Tian et al.: Gliquidone Protects Diabetic Mice from Renal Injury}

\section{Introduction}

Diabetes has become the third most common chronic non-communicable disease threatening human health [1]. One of the frequent complications of diabetes is diabetic nephropathy (DN) [2]. About $40 \%$ of diabetic patients eventually progress to end-stage renal disease (ESRD), and half of these ESRD patients are diagnosed with diabetic nephropathy (DN) [3].

Renal interstitial fibrosis is the ultimate process of diabetic nephropathy, which is characterized by tubular atrophy and interstitial fibrosis [4]. Renal interstitial fibrosis is a common pathway and a major pathological change in the progression of chronic kidney diseases to end-stage renal failure [5]. Currently, studies on renal interstitial fibrosis indicate that more than one-third of interstitial fibroblasts derived from renal epithelial cells through epithelial-mesenchymal transition (EMT). Therefore, EMT might be an important mechanism that leads to renal interstitial fibrosis. EMT of renal tubular epithelial cells is a unique and complicated pathological process which involves a large number of genes, proteins and signaling pathways [6]. Fibrosis of diabetic nephropathy is characterized by accumulation and deposition of extracellular matrix in the mesangial area and tubular interstitium, leading to tubular-interstitial fibrosis, glomerulosclerosis, infiltration of inflammatory cells, and activation of $\alpha$-smooth muscle actin-positive myofibroblasts [7]. Understanding the mechanism of this process will play a positive role in future intervention of diabetic nephropathy.

The Notch pathway has been shown to mediate EMT in epithelial cells in diabetic nephropathy and is associated with activation of TGF- $\beta 1$. The expression of Jagged 1 and hes1 was significantly up-regulated in the kidneys of patients with diabetic nephropathy [8]. In addition, studies have shown that snail can directly inhibit E-cadherin transcription and thus down-regulate its expression, resulting in the loss of epithelial cell adhesion and promotion of EMT. The expression of snail is regulated by a variety of signaling pathways, including Notch signaling pathway. Recent studies have shown that induction of EMT by Notch signaling pathway in renal tissue requires activation of snail molecule, and the expression of Snail1 is directly regulated by Notch signaling [9]. Thus, Notch / Snail pathway is an important pathological mechanism of renal interstitial fibrosis in DN and may be a potential therapeutic target for interstitial fibrosis.

Gliquidone is a second-generation hypoglycemic sulfonylurea that promotes release of endogenous insulin from pancreatic $\beta$-cells and effectively lowers blood glucose [10]. It is rapidly absorbed by the intestinal tract when taken orally and has a biphasic stimulatory effect on insulin secretion. Approximately $95 \%$ of gliquidone is excreted through bile and feces, so drug accumulation is not a problem in patients with renal insufficiency. Therefore, the World Health Organization has recommended it as a first-line drug for the treatment of mild to moderate renal lesions in patients with diabetic nephropathy [11]. In addition to lowering the level of plasma glucose, gliquidone could boost the sensitivities of peripheral tissues and hepatocytes to insulin [12] and enhance the transcription activities of peroxisome proliferator-activated receptor $\gamma$ [13]. Yarat et al. [14] demonstrated that gliquidone markedly lowered the level of nonenzymatic glycosylation protein and total protein and increased the level of lens glutathione in diabetic rats. Yanardag et al. [15] reported that gliquidone lessened the STZ-induced hepatic lesions by reducing oxidative stress in diabetic rats. In patients with diabetic nephropathy, substitution of oral glibenclamide with gliquidone has increased the glomerular filtration rate and lowered urinary albumin [16].

This study examined the effect of gliquidone on the process of EMT in renal tubular epithelial cells in mice with diabetic nephropathy. The role of Notch / Snail1 signaling pathway in inducing EMT differentiation of renal tubular epithelial cells and its association with gliquidone treatment was explored. The results will provide novel ideas for the treatment of diabetic nephropathy in the future. 


\section{Cellular Physiology Cell Physiol Biochem 2018;51:2085-2097 \begin{tabular}{ll|l} 
and Biochemistry Published online: 6 December 2018 & $\begin{array}{l}\text { (c) } 2018 \text { The Author(s). Published by S. Karger AG, Basel } \\
\text { www.karger.com/cpb }\end{array}$ \\
\hline
\end{tabular}

\section{Materials and Methods}

\section{Animals}

Sixty healthy KKAy and ten C57BI / 6J female mice (all SPF grade, 8-10 weeks old) were purchased from Beijing Huafukang Bioscience Co., Inc. Mice were housed in laboratory animal facility with ambient temperature $23-25^{\circ} \mathrm{C}$, relative humidity $50-60 \%$ and $12 \mathrm{~h}$ light/dark cycle. They were provided with free access to food and water. All animal treatments in this study were conducted in accordance with international ethical guidelines on the care and use of laboratory animal, and approved by the Ethics Committee of Shenzhen Third People's Hospital.

\section{Diabetic nephropathy modeling}

One week after the adaptive feeding, the normal feed of KKAy mice was replaced by the special high fat compound feed. The C57BL / 6J mice were continuously fed with normal feed. After 10 weeks of induction feeding, the blood glucose level of all mice was measured, and 24-hour urine was collected using a mousespecific metabolic cage. Mice with intravenous glucose level greater than or equal to $16.7 \mathrm{mmol} / \mathrm{L}$ and $24 \mathrm{~h}$ urine protein $\geq 0.4 \mathrm{mg}$ were considered to be successful model of diabetic nephropathy.

\section{Animal grouping}

The model mice were randomly divided into 5 groups: model group, irbesartan group, low - , middle -, and high- dose gliquidone group, with 10 mice in each group. The irbesartan group was intra-gastrically administered with irbesartan at $25 \mathrm{mg} / \mathrm{kg} / \mathrm{d}$, which was 10 times the dose of clinical patients. The doses of gliquidone in low-, medium- and high- dose groups were 3.5, 7 and 14 times higher than the dose of clinical patients $(0.525 \mathrm{~g} / \mathrm{kg} / \mathrm{d}, 1.05 \mathrm{~g} / \mathrm{kg} / \mathrm{d}$ and $2.1 \mathrm{~g} / \mathrm{kg} / \mathrm{d}$ ). All C57bl / 6J mice were normal control group. Mice in normal control group and model group were administered with an equal volume of deionized water $(0.1 \mathrm{ml} / 10 \mathrm{~g}$ body weight). Gastric gavage was given once daily for 16 weeks. The mental status, activity, changes in fur and limbs, urinary and genital conditions, changes in water intake and body weight were observed and recorded every four weeks.

\section{Urinary protein quantitation}

The $24 \mathrm{~h}$ urine of mice was collected on the $10 \mathrm{th}, 14 \mathrm{th}, 18 \mathrm{th}, 22 \mathrm{th}$ and 26 th weeks after the start of drug administration. The $24 \mathrm{~h}$ urinary protein content was extracted using liquid protein extraction kit-II (P1255, Applygen Technologies Inc., Beijing, China), and urine protein concentration measured by BCA kit (P1511, Applygen Technologies Inc., Beijing, China). Combined with 24h urine output, 24h urinary protein was calculated. The procedures were performed according to the manufacturer's instructions.

\section{Kidney weight and body weight ratio of mice}

After the body weight was measured at week 26, the mice were anesthetized by intraperitoneal injection of $4 \%$ chloral hydrate $(0.1 \mathrm{ml} / 10 \mathrm{~g})$. The kidneys were collected and weighed. The ratio of kidney weight to body weight was calculated. Then the left kidney was sliced along the long axis and fixed in 10\% neutral buffered formalin solution. Small pieces of the right kidney tissue were taken, snap frozen in liquid nitrogen and transferred to a $-80^{\circ} \mathrm{C}$ freezer for later use.

\section{Blood collection and serum biochemical index detection}

Blood taken from eyes was used in this experiment. Blood glucose was determined by the OneTouch blood glucose meter (Johnson \& Johnson Medical Ltd, Shanghai). The levels of serum BUN, Scr, TG were detected by an automatic biochemical analyzer (Hitachi 7160 automatic biochemical analyzer, Hitachi, Japan).

\section{Detecting oxidative stress indicators}

Fresh kidney tissue $(0.5 \mathrm{~g}$ ) was thoroughly ground in liquid nitrogen and $10 \mathrm{~mL}$ of potassium phosphate buffer (50 mM, pH 7.5). The homogenate was centrifuged and an aliquot of supernatant was used to determine the content of oxidative stress biomarkers (MDA, NO and SOD), according to the manufacturer's 


\section{Cellular Physiology Cell Physiol Biochem 2018;51:2085-2097 \begin{tabular}{l|l|l} 
and Biochemistry & $\begin{array}{l}\text { DOI: 10.1159/000495827 } \\
\text { Published online: 6 December } 2018\end{array}$ & $\begin{array}{l}\text { @ } 2018 \text { The Author(s). Published by S. Karger AG, Basel } \\
\text { www.karger.com/cpb }\end{array}$ \\
\hline
\end{tabular}}

instructions in MDA/NO/SOD kits (MDA kit: HY-60003; NO kit: HY-60018; SOD kit: HY-60001, Beijing Sinouk institute of Biological Technology, Beijing, China). Samples were put into the automatic biochemical analyzer (Hitachi 7160 automatic biochemical analyzer, Hitachi, Japan) and the MDA, NO and SOD content were read directly.

\section{RNA extraction and quantitative real-time PCR ( $q R T-P C R)$}

Total RNAs from peripheral blood cells were extracted using TRIzol reagent (Invitrogen). The relative expression was determined by using the mirVana qRT-PCR detection kit (Thermo Fisher Scientific) in accordance with the manufacturer's instructions. The relative mRNA expressions of $\alpha$-SMA and E-cadherin were detected using the standard SYBR-Green real-time PCR kit (Takara, Dalian, China). GAPDH was used as an internal reference. Primer sequences are given in Supplementary Table S1 (for all supplementary material see www.karger.com/doi/ 10.1159/000495827). The relative expression levels were quantified using the comparative Ct method.

\section{Western Blotting}

A bicinchoninic acid (BCA) kit (Sigma-Aldrich, St. Louis, USA) was used to determine the protein concentration. Proteins (30 $\mu \mathrm{g}$ per sample per well) were added and separated in $10 \%$ polyacrylamide gel electrophoresis. After electrophoresis, proteins were transferred to polyvinylidene fluoride (PVDF) membranes with $100 \mathrm{~V}$ transfer-molded voltage for 45 to $70 \mathrm{~min}$. Then, samples were incubated at room temperature for $1 \mathrm{~h}$ with 5\% bovine serum albumin (BSA), and then with primary antibodies (1: 1000 dilution) at $4^{\circ} \mathrm{C}$ overnight. After that, samples were washed with tris-buffered saline Tween 20 (TBST) 3 times ( $5 \mathrm{~min} /$ time). The corresponding secondary antibodies were added for incubation at room temperature for $1 \mathrm{~h}$. After that, membranes were washed 3 times ( $5 \mathrm{~min} /$ time). Development was completed with chemiluminescence reagents. GAPDH was used as an internal reference. Bands were visualized with a Bio-Rad Gel Doc EZ imager (Life Science Research, California, USA). Image J software (National Institutes of Health, Maryland) was applied to analyze the intensity of the target bands.

\section{Statistical analysis}

All data were analyzed by GraphPad Prism version 6 statistical software. Measurement data were expressed by mean \pm standard deviation. The $t$ test was used for comparisons between two groups. Oneway analysis of variance was applied for comparisons between multiple groups. Statistical significance was assumed for $P<0.05$.

\section{Results}

Effect of gliquidone on general health condition of mice with diabetic nephropathy

After KKAy mice were fed with special feed, mice appeared apathetic, with reduced activity and fur luster, as well as significantly increased water consumption, compared with C57bl / 6J mice. The 24h urine output significantly increased. After ten weeks of special feed, diabetic complications such as skin ulcers and urinary tract infections occurred in mice with no drug treatment. Compared with the model group, the mice in irbesartan and gliquidone treatment groups had improved general health conditions, such as better mental status, better fur, better eating and drinking and more activity. This result indicated that gliquidone had no toxic effect on mice and

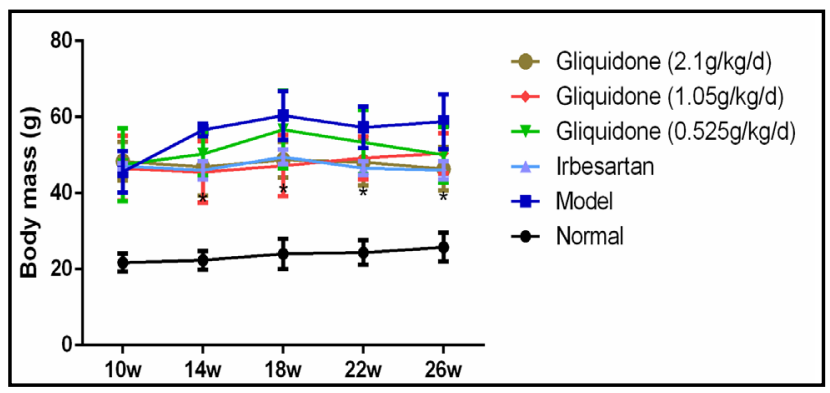

Fig. 1. Effect of gliquidone on body mass in KKAy mice. Model mice had significantly higher body mass than other mice. Gliquidone and Irbesartan treatments significantly decrease body mass of mice $(*$ indicates that $\mathrm{P}<0.05$ compared to Model group). 
can improve the general health condition of diabetic mice.

\section{Gliquidone lowered body weight of mice with diabetic nephropathy \\ The body weight of KKAy mice} with diabetic nephropathy gradually increased at the beginning of gliquidone treatment (Fig. 1). There was a significant difference in body weight $(P<0.01)$ between diabetic nephropathy mice and C57bl / 6J mice. As the increase in body weight of mice in treatment groups slowed down with time, the body weight of mice in each treatment group significantly differed from that of the model group from the 14th week $(P<0.01)$. At week 18, there was a significant difference between the low-dose gliquidone and irbesartan groups $(P<0.01)$. As treatment time increased, there was no significant difference of body weight between the treatment groups $(P>0.05)$. However, the average body weight in high-dose gliquidone group was significantly lower than that of lowor medium- dose treatment groups.

Effect of gliquidone on renal weight to body weight ratio in mice with diabetic nephropathy

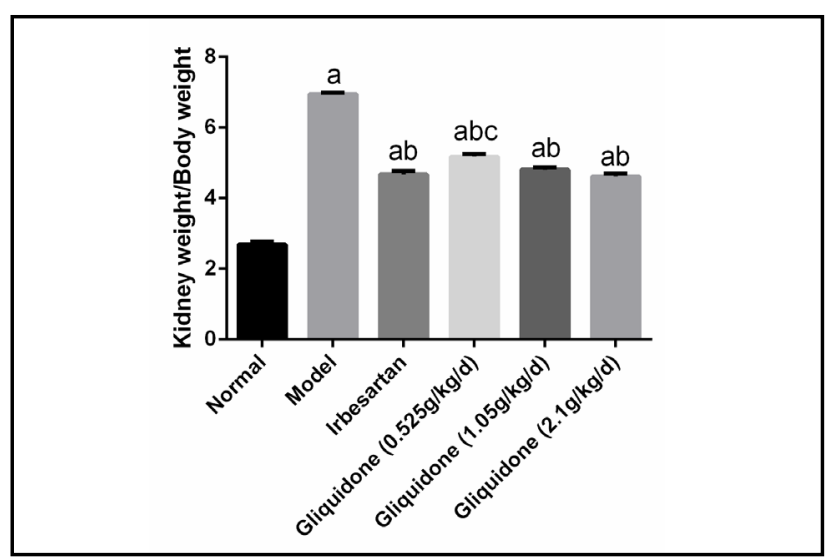

Fig. 2. Effect of gliquidone on Kidney Weight to Body Weight Ratio in KKAy mice (a indicates $\mathrm{P}<0.05$ compared to normal group, b indicates $\mathrm{P}<0.05$ compared to model group, $\mathrm{c}$ indicates $\mathrm{P}<0.05$ compared to Irbesartan group).

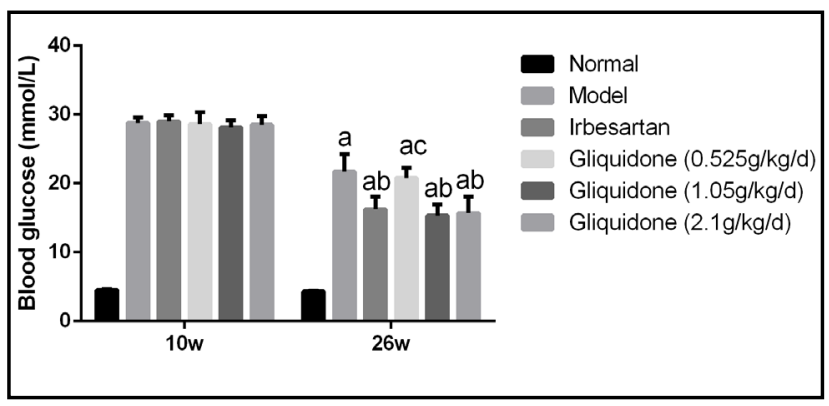

Fig. 3. Effect of gliquidone on blood glucose in KKAy mice (a indicates $\mathrm{P}<0.05$ compared to normal group, $\mathrm{b}$ indicates $\mathrm{P}<0.05$ compared to model group, $\mathrm{c}$ indicates $\mathrm{P}<0.05$ compared to Irbesartan group).

After 16 weeks of treatment, the ratio of kidney weight to body weight (KW / BW) in mice with diabetic nephropathy was significantly higher than that in each treatment group and normal control group $(P<0.01)$. There was no significant difference of KW / BW ratio between high- and medium dose gliquidone groups $(P>0.05)$. There was a significant difference of KW / BW ratio between low- and medium- dose gliquidone groups $(P<0.05)$, and between high- and low- dose gliquidone groups $(P<0.01)$. There was no significant difference of KW / BW ratio between medium- or high- dose gliquidone group and irbesartan group $(P>0.05)$. There was a significant difference of KW / BW ratio between gliquidone low dose group and irbesartan group $(P<0.05)$. Numerically, the effect of the high-dose gliquidone group was most pronounced (Fig. 2).

\section{Gliquidone lowered blood glucose of mice with diabetic nephropathy}

The blood glucose level of KKAy mice was measured at 10 weeks to be over $16.8 \mathrm{mmol}$ / L, which met the diagnostic criteria of diabetes mellitus. After 16 weeks of intervention, the blood glucose concentrations were measured again. The results showed that the blood glucose levels of mice in each treatment group decreased, except for the low-dose gliquidone group. The differences in blood glucose level for each treatment group was statistically significant compared with the model group $(P<0.05)$. There was no significant difference of blood glucose level among gliquidone high-, or medium- dose group and irbesartan group (Fig. 3) $(P>0.05)$. 
Effect of gliquidone on blood fats in mice with diabetic nephropathy

As shown in Fig. 4C, the blood fats in the model group at 26 weeks of age were significantly higher than those in the normal mice group $(\mathrm{P}<0.05)$, and were significantly higher than those in the other groups $(\mathrm{P}<0.05)$. Among them, the group with anti-hyperlipidemic drug irbesartan was the most obvious. The blood lipid of irbesartan group was lower than that of the low/ medium dose gliquidone group $(\mathrm{P}<0.05)$. There was no significant difference in blood fats in gliquidone high, medium and low dose groups $(\mathrm{P}>0.05)$.

\section{Gliquidone improved renal function in mice with diabetic nephropathy}

At week 26, serum creatinine (Scr, Fig. 4B) and blood urea nitrogen (BUN, Fig. 4A) were significantly increased in KKAy mice $(\mathrm{P}<0.05)$ compared with those in normal group. After 16 weeks of intra-gastric administration of gliquidone, the levels of Scr in all treatment groups were significantly lower than those in model group $(\mathrm{P}<0.05)$ except for the low dose and medium dose gliquidone group. Meanwhile, the levels of BUN in each treatment groups were significantly lower than those in the model group $(\mathrm{P}<0.05)$. The levels of BUN in irbesartan group were not significantly different from those of the gliquidone groups $(P>0.05)$. These results indicate that, similarly to irbesartan, gliquidone can improve renal function of mice with diabetic nephropathy.

\section{Gliquidone lowered 24 h urine protein in mice with diabetic nephropathy}

As shown in Fig. 5, the urinary protein content of model group gradually increased over time, and was significantly higher than that of the control group $(\mathrm{P}<0.01)$ at 14 weeks. Compared with the model group, the $24 \mathrm{~h}$ urinary protein content of each treatment group was significantly decreased $(\mathrm{P}<0.01)$. The urine protein of mice in each treatment group did not increase significantly over time, and the average urinary protein levels in mice treated with gliquidone were significantly lower than those in the model group at weeks 18,22 and $26(\mathrm{P}<0.01)$. Urinary protein in the 26th week was significantly lower than that in the previous period. Urinary protein in the high dose gliquidone group decreased most significantly compared with the model group, and there was a significant difference compared with the irbesartan group $(\mathrm{P}<0.05)$.

\section{Effect of gliquidone on oxidative stress in kidneys of mice with diabetic nephropathy}

The level of MDA in the model group was significantly higher than that in the normal control group $(P<0.05)$. The MDA level in each treatment group was significantly lower than that in the model group ( $\mathrm{P}<0.01)$. There was no significant difference of MDA level in gliquidone high or medium dose groups $(\mathrm{P}>0.05)$. Low dose gliquidone group had significantly higher MDA level than that in the high dose group $(\mathrm{P}<0.05)$ (Fig. 6A).

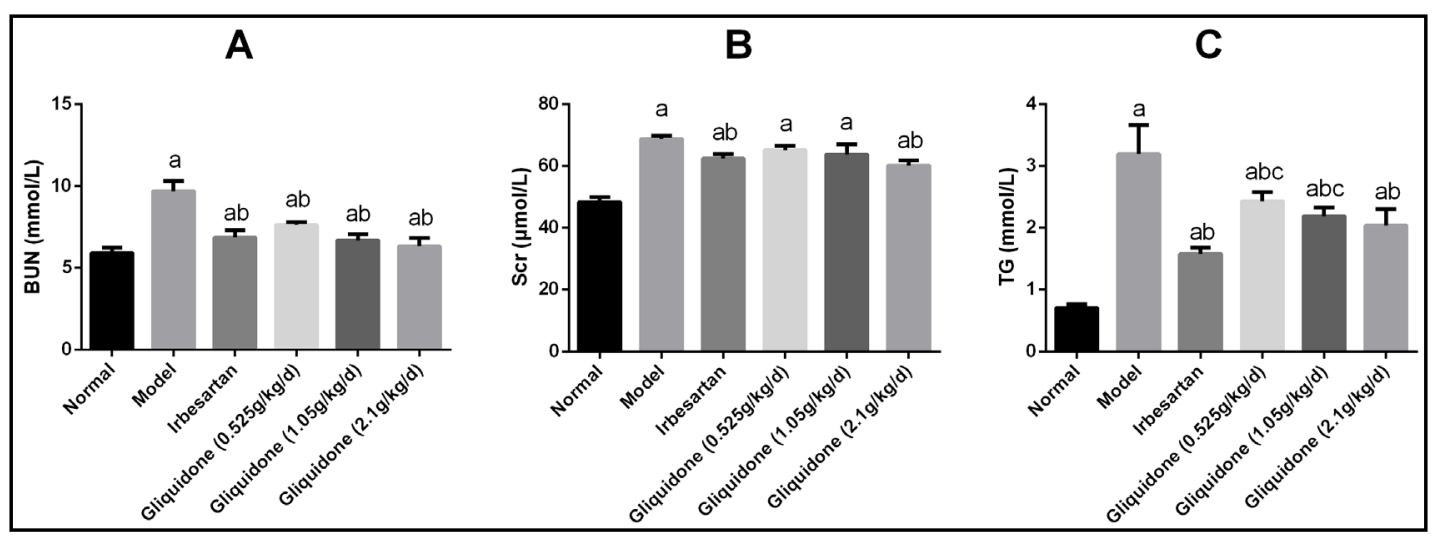

Fig. 4. Effect of gliquidone on BUN, Scr, and TG in KKAy mice (A: Blood urea nitrogen concentration; B: Serem creatinine concentration; C: Triglyceride concentration. a indicates $\mathrm{P}<0.05$ compared to normal group, b indicates $\mathrm{P}<0.05$ compared to model group, $\mathrm{c}$ indicates $\mathrm{P}<0.05$ compared to Irbesartan group). 
The level of NO in model group was significantly lower than that in normal control group $(P<0.05)$. The NO level in each treatment group was significantly higher than that in model group $(\mathrm{P}<0.01)$. There was no significant difference in NO levels between gliquidone high and medium dose groups $(\mathrm{P}>0.05)$. The level of $\mathrm{NO}$ in the low-dose gliquidone group was significantly lower than that in the high-dose group ( $\mathrm{P}<0.05$ ) (Fig. 6B).

The level of SOD in the model group was significantly lower than that in the normal control group $(P<0.05)$. The SOD level in each treatment group was significantly higher than that in the model group $(\mathrm{P}<0.01)$. The level of SOD in gliquidone medium and low dose groups was significantly lower than that in Irbesartan group $(\mathrm{P}<0.05)$. There was no significant difference in SOD level between gliquidoctone medium and low dose groups ( $\mathrm{P}>$ 0.05) (Fig. 6C).

qRT-PCR and immunohistochemistry on the expression of $m R N A$ and protein of $\alpha$-SMA and E-cadherin in mice with diabetic nephropathy

The expression of a-SMA mRNA in renal tubular epithelial cells in model group was significantly increased compared with normal C57bl / 6J mice $(\mathrm{P}<0.01)$. The expression of $\alpha$-SMA mRNA in renal tubular epithelial cells in each treatment group decreased compared with the model group. There was no statistical difference in $\alpha$-SMA mRNA expression between the high-dose and middle-dose gliquidone groups $(\mathrm{P}>0.05)$. Expression of E-cadherin mRNA in renal tubular epithelial cells significantly decreased in the model group compared with normal $\mathrm{C} 57 \mathrm{bl} / 6 \mathrm{~J}$ mice $(\mathrm{P}<0.01)$. E-cadherin mRNA expression in renal tubular epithelial cells of mice in each treatment group increased compared with the model group. There

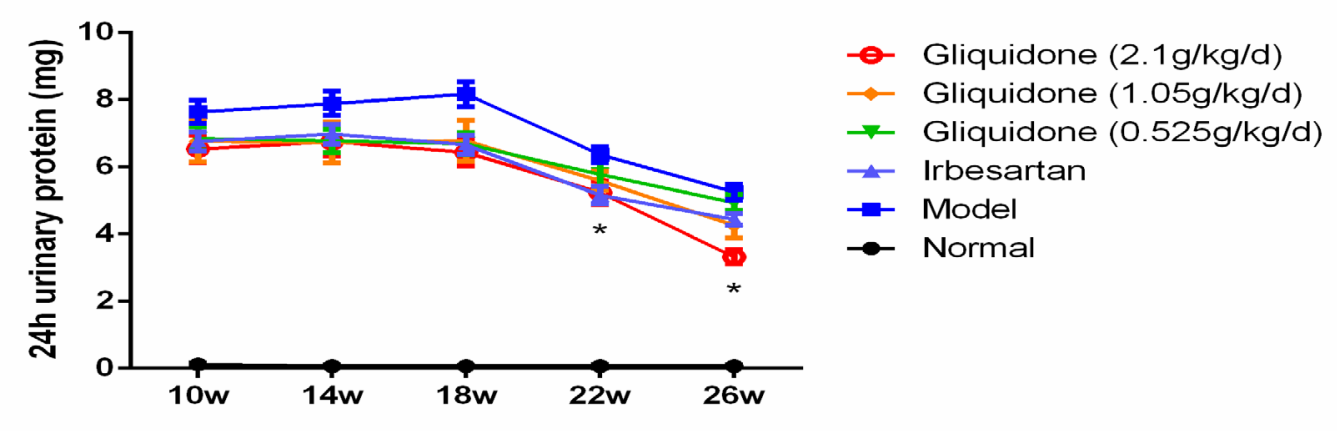

Fig. 5. Effect of gliquidone on urinary protein in KKAy mice $(*$ indicates that $\mathrm{P}<0.05$ compared to Model group).

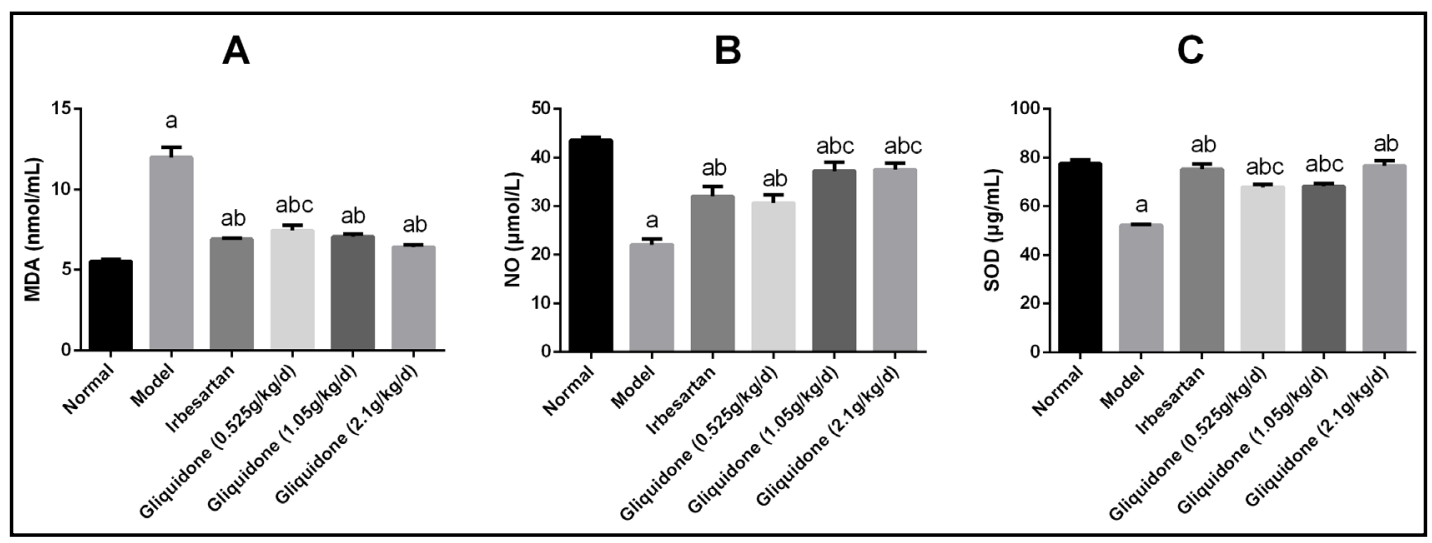

Fig. 6. Effect of gliquidone on oxidative stress indexes in KKAy mice (A: Malondialdehyde concentration; B: Nitric oxide concentration; C: Superoxide dismutase concentration. a indicates $\mathrm{P}<0.05$ compared to normal group, b indicates $\mathrm{P}<0.05$ compared to model group, $\mathrm{c}$ indicates $\mathrm{P}<0.05$ compared to Irbesartan group). 
was no significant difference between high-dose gliquidone and irbesartan group, and no significant difference between medium-dose and low-dose gliquidone group (all $\mathrm{P}>0.05$ ). Expression of E-cadherin mRNA in high-dose group was significantly higher than that in medium- and low-dose groups $(\mathrm{P}<0.05)$ (Fig. 7A).

The expression of $\alpha$-SMA protein in tubular epithelial cells of $\mathrm{C} 57 \mathrm{bl} / 6 \mathrm{~J}$ mice in normal control group was low. The expression of $\alpha$-SMA protein in tubular epithelial cells in the model group increased significantly compared with the normal group $(P<0.05)$. The expression of $\alpha$-SMA protein in renal tubular epithelial cells in each treatment group was significantly reduced compared with the model group, while the expression of $\alpha$-SMA protein in the lowdose gliquidone group was significantly higher than those of irbesartan group and gliquidone high-dose group $(P<0.05)$. There was no significant difference of $\alpha$-SMA protein expression in Irbesartan group and gliquidone high- or medium- dose group $(\mathrm{P}>0.05)$. Expression of E-cadherin protein was higher in $\mathrm{C} 57 \mathrm{bl} / 6 \mathrm{~J}$ mice than in model mice. E-cadherin protein expression in renal tubular epithelial cells in each treatment group significantly increased, while expression of E-cadherin in low dose gliquidone group was significantly lower than that in irbesartan group and high dose gliquidone group (all $\mathrm{P}<0.01$ ). There was a significant difference of E-cadherin protein expression between high-dose and low-dose gliquidone groups $(\mathrm{P}<0.05)$ (Fig. 7B).

The expression of Jagged1, Notch1, hes1 and Snail1 protein in renal tissues of mice with diabetic nephropathy

The expression of Jagged 1 protein in the model group significantly increased compared with normal control group (Fig. 8A, $\mathrm{P}<0.01$ ). Jagged1 protein expression in the kidneys of each treatment group was significantly lower than that of the model group $(\mathrm{P}<0.01)$. The level of Jagged1 expression in the high-dose gliquidone and irbesartan groups was not significantly different from that in the normal control group ( $P>0.05)$. However, the expression of Jagged1 in medium and low dose gliquidone groups were significantly higher than that in normal control group $(\mathrm{P}<0.05)$. The expression of Notch1 protein (Fig. 8B) was similar to that of Jagged1, but the expression of Notch1 in irbesartan group and high-dose gliquidone group was significantly higher than that in normal control group (P $<0.05$ ). The expression of hes1 protein (Fig. 8C) was generally similar to that of Jagged1, but there was no significant difference between control group and high-dose gliquidone group. The expression of hes1 in medium-dose gliquidone group and irbesartan group was not significantly different $(\mathrm{P}>0.05)$. The expression of Snail1 protein (Fig. 8D) was similar to that of hes1, but the expression of high dose gliquidone group was significantly higher than that of normal control group $(\mathrm{P}<0.01)$.

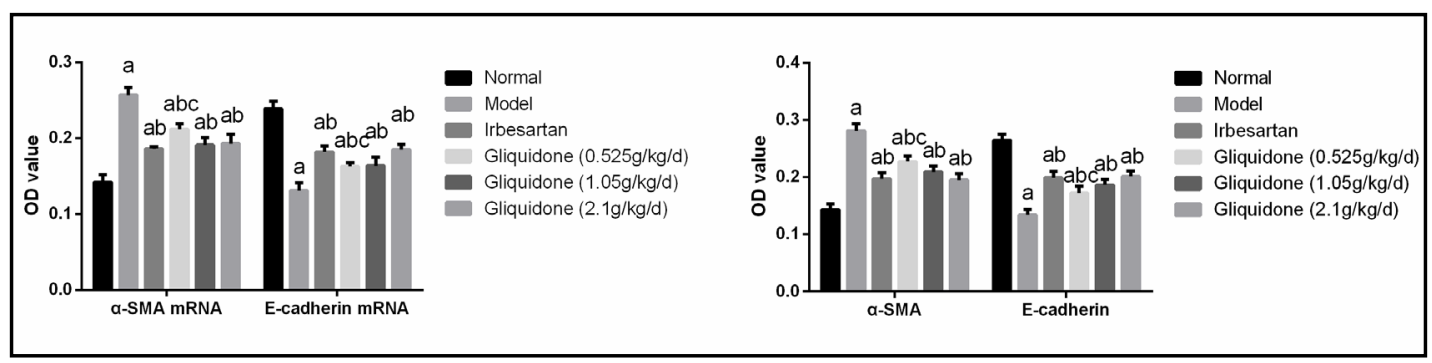

Fig. 7. Effect of gliquidone on EMT in KKAy mice (Left: qRT-PCR results showing mRNA expression of each group; Right: immunohistochemistry results showing protein expression of each group. a indicates $\mathrm{P}<0.05$ compared to normal group, $\mathrm{b}$ indicates $\mathrm{P}<0.05$ compared to model group, $\mathrm{c}$ indicates $\mathrm{P}<0.05$ compared to Irbesartan group).

\section{KARGER}




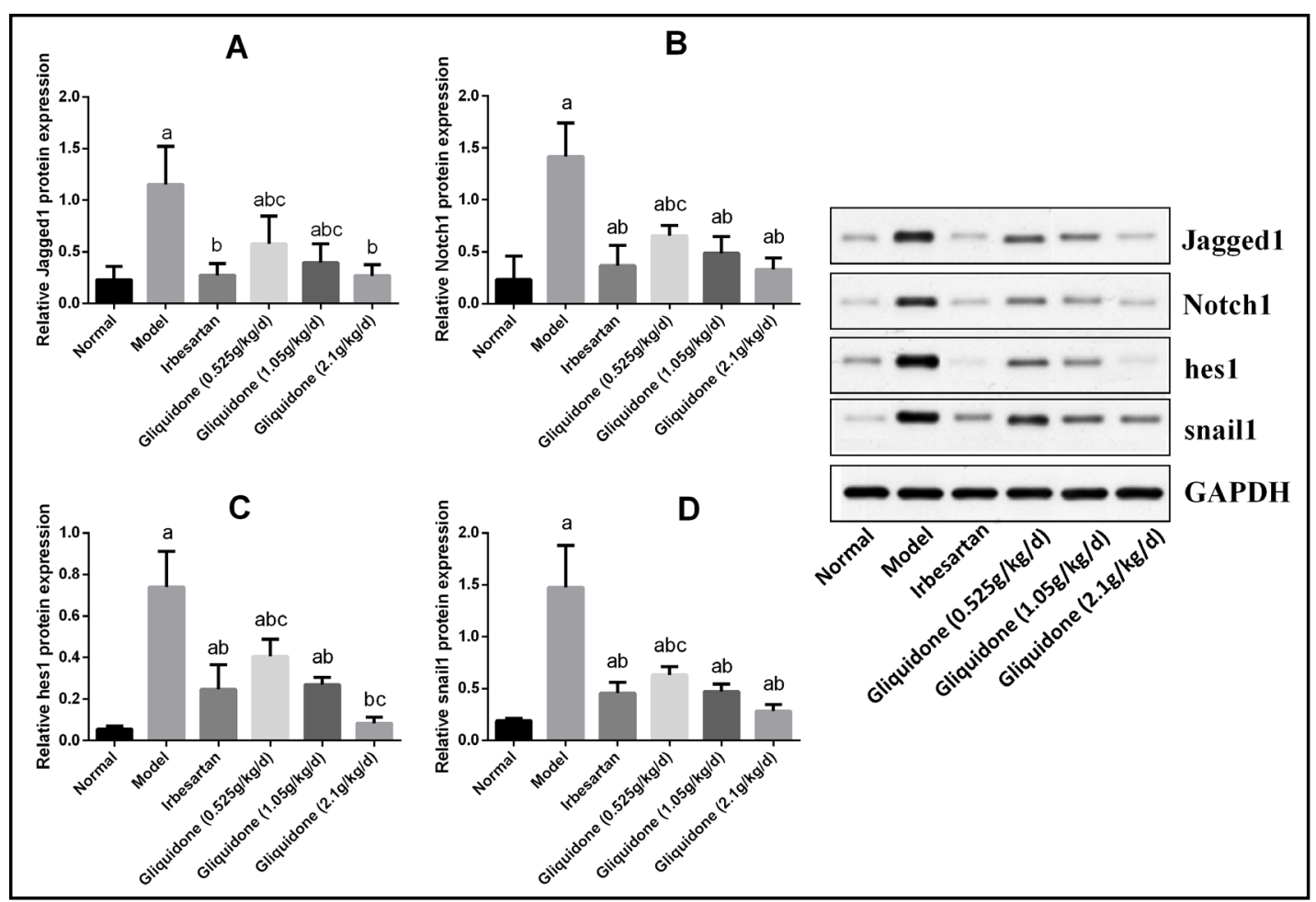

Fig. 8. Effect of gliquidone on Notch/Snail pathway in KKAy mice (A: Jagged1 protein expression; B: Notch1 protein expression; C: hes1 protein expression; D: Snail1 protein expression. a indicates $\mathrm{P}<0.05$ compared to normal group, $\mathrm{b}$ indicates $\mathrm{P}<0.05$ compared to model group, $\mathrm{c}$ indicates $\mathrm{P}<0.05$ compared to Irbesartan group).

\section{Discussion}

The incidence of diabetic nephropathy is increasing because of the socio-economic development and changes in lifestyle and diet in China. Currently about $7 \%$ of Chinese adults are diabetic, and about one-third of diabetic patients with 10 to 20 years of history develop diabetic nephropathy. The patient population of diabetic nephropathy in China tends to be younger. Ninety-two percent of diabetic patients eventually develop diabetic complications, and diabetic nephropathy is one of the major causes of chronic renal failure [17]. The treatment of diabetic nephropathy is much more complicated than general kidney diseases and its prognosis is poor. The pathogenesis of diabetic nephropathy is poorly understood and there is no effective prevention and treatment for it.

In this study, KKAy mice were fed with high-fat diet to establish an end-stage diabetic nephropathy mouse model. Both fat and lean tissue increased in KKAy diabetic mice compared to normal mice, with fat accounting for $30-35 \%$ of total body weight. This mouse model has many advantages, including small individual differences, short formation time, and good reproducibility. Its pathogenesis is similar to that of human type 2 diabetes mellitus [18]. Studies have shown that blood glucose and urinary protein excretion increased significantly in KKAy diabetic mice after 8 weeks on a high-fat diet. Kidney weight and body weight was significantly increased in KKAy diabetic mice compared with that of the control mice. Compared with the normal control group, the body weight, blood glucose and renal function indices (serum creatinine and urea nitrogen et al.) in the model group were significantly increased, and the $24 \mathrm{~h}$ urinary protein excretion increased gradually with time. This shows that the diabetic mouse model was successfully established. The drug treatment improved the renal interstitial fibrosis, with the gliquidone high-dose group exhibiting a particularly prominent effect, similar to the curative effect of positive control drug irbesartan. 


\section{Cellular Physiology Cell Physiol Biochem 2018;51:2085-2097

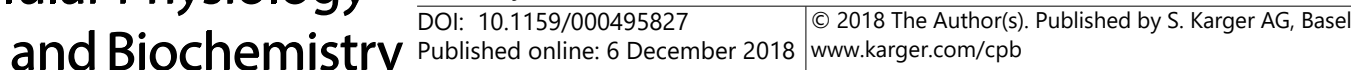 \\ Tian et al.: Gliquidone Protects Diabetic Mice from Renal Injury}

The main pathological changes in end-stage renal failure of chronic nephropathy are the appearance of renal interstitial fibrosis [19]. Studies found that more than $30 \%$ of stromal fibroblasts come from tubular epithelial cells through EMT process [20]. A large number of studies showed that many cells in the body could stimulate EMT process under certain conditions [21]. Tubular epithelial cells have strong plasticity, which allows them to adapt to different living environments and make appropriate self-regulatory responses to different external stimuli. EMT have 3 biological subtypes [22]: EMT type 1 is primarily involved in embryogenesis and organ development, and the resulting mesenchymal stem cells are an important source of tissue and organ formation. This process is short and generally does not result in fibrosis; Type 2 EMT is associated with injury repair. It is generally accepted that cells undergo fibroblast transformation through the EMT process in post-injury selfrepair. The gradual accumulation of fibroblasts, under the action of sustained damage and inflammation, leads to development of fibrosis. The massive accumulation of fibroblasts eventually results in destruction of organ structure and function; Type 3 EMT is involved in tumor invasion and migration. The occurrence of renal tissue EMT is a complicated process with many steps. A variety of pathological factors can lead to renal tubular epithelial cell damage. Under continuous stimulation of harmful factors, the expression of epithelial adhesion molecules (E-cadherin) is reduced, the expression of fibroblast markers ( $\alpha$-SMA) is increased, and protease is activated to dissolve the tubular basement membrane, leading to tubulointerstitial fibrosis. Thus, inhibition of renal tubular epithelial cell differentiation may be an important step in delaying the progression of diabetic nephropathy. E-cadherin is the most classic epithelial cell marker. Loss of E-cadherin is a sign of epithelial cell transdifferentiation, and the loss of E-cadherin can further promote the development of EMT, eventually leading to trans-differentiation of epithelial cells into fibroblasts, resulting in tubulointerstitial fibrosis. Alpha-smooth muscle actin is one of the six actin families that is abundantly expressed in vascular smooth muscle cells and myoepithelial cells and is also one of the markers for type 2 EMT [23]. Therefore, the detection of changes of E-cadherin and $\alpha$-SMA in kidney tissue of mice with diabetic nephropathy indicate the involvement of EMT process in renal tubular epithelial cells. In this study, qRT-PCR and immunoblotting were used to detect the expression of E-cadherin and $\alpha$-SMA in mice with diabetic nephropathy. The results showed that the protein and mRNA expression of E-cadherin in the late stage of KKAy mice was significantly decreased and the protein and mRNA expression of $\alpha$-SMA was significantly increased. After intervention with different doses of gliquidone, the protein and mRNA expression of E-cadherin increased, and the protein and mRNA expression of $\alpha$-SMA decreased significantly compared with the model group.

Notch signaling pathway consists of Notch receptor, Notch ligand (DSL protein), CSL (CBF-1, Suppressor of hairless, Lag) DNA binding protein, and other effectors and regulatory elements of Notch. Mammals have 4 Notch receptors and 5 Notch ligands. Notch ligands from adjacent cells bind to their counterparts, and after three enzymatic cleavages, they are released into the cytoplasm and nucleus, and bind to the gene of interest to form a transcriptional activation complex that activates the target gene of the transcriptional repressor family (HES , HEY, HERP and other basic-helix-loop-helix (bHLH)) [24]. Notch signaling pathway is involved in regulation of various diseases, including the majority of kidney diseases and glomerular diseases. Studies have shown that Notch signaling pathway is also involved in the regulation of cell proliferation and apoptosis. Morrisey et al. reported [25] that TGF- $\beta 1$ can up-regulate the expression of renal Jagged1 / Notch signaling pathway to mediate renal fibrosis in mice. In addition, experiments have confirmed that the expression of Jagged1, Notch2 and Notch4 is necessary for TGF- $\beta 1$-induced EMT. Murea et al. [26] found that activation of the Notch pathway is a common pathogenesis of proteinuric kidney disease and is prevalent in the pathophysiology of glomerulosclerosis and tubulointerstitial fibrosis. Walsh et al. [27] found that the expression of Jagged1 and Hes1 in renal tubules of interstitial fibrosis in patients with diabetic nephropathy was significantly up-regulated. The above studies suggest that the regulation of Notch1 / Jagged1 signaling pathway and TGF- $\beta$ 


\section{Cellular Physiology Cell Physiol Biochem 2018;51:2085-2097 \begin{tabular}{ll|l} 
DOI: 10.1159/000495827 & $\begin{array}{l}\text { O } 2018 \text { The Author(s). Published by S. Karger AG, Basel } \\
\text { www.karger.com/cpb }\end{array}$ \\
\hline and Biochemistry
\end{tabular}

may play a key role in the progression of diabetic nephropathy, which fully demonstrates the importance of Notch pathway in EMT progression of diabetic nephropathy.

Members of the zinc finger transcription factor superfamily, which includes snail and slug genes, can inhibit gene transcription by binding to the E-box structure [28]. Studies found that snail can directly inhibit E-cadherin transcription and thus down-regulate its expression, which can in turn lead to loss of adhesion between epithelial cells and promotion of EMT. This process is very common in embryogenesis, tissue fibrosis and tumor invasion [29]. Studies have shown that TGF- $\beta 1$ induced a significant increase in snail expression in human renal epithelial cells, accompanied by $\alpha$-SMA up-regulation. These results demonstrated that snail is involved in the EMT process of human renal tubular epithelial cells [30]. The promotion of EMT in renal tubular epithelial cells by snail is regulated by many upstream signaling pathways, and Notch signaling pathway is one of them [9]. It was shown that activation of Notch2 and Notch4 promotes the activation of Snail. N2ICD and N4ICD overexpression can induce snail upregulation, and snail expression is directly regulated by Notch signaling pathway [31].

In this study, we showed that the expression of Jagged1, Notch1, Hes1 and Snail1 in the model group were significantly increased compared with the normal group, and the levels of these indices were significantly reduced after treatment with gliquidone, indicating that gliquidone.can effectively block the activation of the Notch / Snail signaling pathway. These results indicated that Jagged / Notch signaling pathway and snail molecule are important in inducing renal interstitial fibrosis in diabetic nephropathy, which is important for the intervention of renal interstitial fibrosis in diabetic nephropathy. This study provides a new direction for using drug targeting to delay the progression of diabetic nephropathy.

\section{Conclusion}

Taken together, our results suggest that gliquidone is effective in inhibiting EMT progression and renal interstitial fibrosis of diabetic nephropathy through blocking the Notch / snail signaling pathway in a dose-dependent manner, delaying the onset of diabetic nephropathy.

\section{Acknowledgements}

This work was supported by Shenzhen Science and Technology Innovation Committee (JCYJ20170307094746491).

\section{Disclosure Statement}

The authors declare no conflict of interest.

\section{References}

1 Shi Y, Hu FB: The global implications of diabetes and cancer. Lancet 2014;383:1947-1948.

2 Parveen A, Jin M, Kim SY: Bioactive phytochemicals that regulate the cellular processes involved in diabetic nephropathy. Phytomedicine 2018;39:146-159.

-3 Weir MA, Herzog CA: Beta blockers in patients with end-stage renal disease-Evidence-based recommendations. Semin Dial 2018;10.1111/sdi.12691.

4 Wu Y, Wang L, Wang X, Wang Y, Zhang Q, Liu W: Renalase contributes to protection against renal fibrosis via inhibiting oxidative stress in rats. Int Urol Nephrol 2018;10.1007/s11255-018-1820-2. 


\section{Cellular Physiology Cell Physiol Biochem 2018;51:2085-2097 \begin{tabular}{l|l|l} 
DOI: 10.1159/000495827 & (c) 2018 The Author(s). Published by S. Karger AG, Basel
\end{tabular} and Biochemistry Published online: 6 December 2018 www.karger.com/cpb}

5 Cheng Z, Liu L, Wang Z, Cai Y, Xu Q, Chen P: Hypoxia Activates Src and Promotes Endocytosis Which Decreases MMP-2 Activity and Aggravates Renal Interstitial Fibrosis. Int J Mol Sci 2018;19:E581.

-6 Qi FH, Cai PP, Liu X, Si GM: Adenovirus-mediated P311 ameliorates renal fibrosis through inhibition of epithelial-mesenchymal transition via TGF-beta1-Smad-ILK pathway in unilateral ureteral obstruction rats. Int J Mol Med 2018;10.3892/ijmm.2018.3485.

7 Li X, Xu L, Hou X, Geng J, Tian J, Liu X, Bai X: Advanced oxidation protein products aggravate tubulointerstitial fibrosis through PKC-dependent mitochondrial injury in early diabetic nephropathy. Antioxid Redox Signal 2018;10.1089/ars.2017.7208.

-8 Yang G, Zhao Z, Zhang X, Wu A, Huang Y, Miao Y, Yang M: Effect of berberine on the renal tubular epithelialto-mesenchymal transition by inhibition of the Notch/snail pathway in diabetic nephropathy model KKAy mice. Drug Des Devel Ther 2017;11:1065-1079.

-9 Simi AK, Anlas AA, Stallings-Mann M, Zhang S, Hsia T, Cichon MA, Radisky DC, Nelson CM: A soft microenvironment protects from failure of midbody abscission and multinucleation downstream of the EMT-promoting transcription factor Snail. Cancer Res 2018;10.1158/0008-5472.CAN-17-2899.

10 Liu SY, Tian HM, Liao DQ Chen YF, Gou ZP, Xie XY, Li XJ: The effect of gliquidone on KATP channels in pancreatic beta-cells, cardiomyocytes, and vascular smooth muscle cells. Diabetes Res Clin Pract 2015;109:334-339.

11 Tan Z, Xu Z, Gui Q, Wu W, Yang Y: Gliquidone versus metformin: differential effects on aorta in streptozotocin induced diabetic rats. Chin Med J (Engl) 2014;127:1298-1303.

12 Malaisse WJ: Gliquidone contributes to improvement of type 2 diabetes mellitus management: a review of pharmacokinetic and clinical trial data. Drugs R D 2006; 7:331-337.

$>13$ Lee KW, Ku YH, Kim M, Ahn BY, Chung SS, Park KS: Effects of Sulfonylureas on Peroxisome ProliferatorActivated Receptor gamma Activity and on Glucose Uptake by Thiazolidinediones. Diabetes Metab J 2011;35:340-347.

14 Yarat A, Tunali T, Yanardag R, Gursoy FO, Sacan 00, Emekli N, Utuner A, Ergenekon G: The effect of Glurenorm (gliquidone) on lenses and skin in experimental diabetes. Free Radic Biol Med 2001;31:10381042.

15 Yanardag R, Ozsoy-Sacan 0, Orak H, Ozgey Y: Protective effects of glurenorm (gliquidone) treatment on the liver injury of experimental diabetes. Drug Chem Toxicol 2005;28:483-497.

-16 Mazurov VI, Novik AA, Nagibovich OA, Romashevskii BV, Guliaeva IV, Kurganova TA: [Effect of sugarreducing therapy on renal function in patients with type II diabetes mellitus]. Klin Med (Mosk) 1998;76:3841.

17 Yu X, Chau JPC, Huo L: The effectiveness of traditional Chinese medicine-based lifestyle interventions on biomedical, psychosocial, and behavioral outcomes in individuals with type 2 diabetes: A systematic review with meta-analysis. Int J Nurs Stud 2018;80:165-180.

18 Gao Y, Gu C, Wang K, Wang H, Ruan K, Xu Z, Feng Y: The effects of hypoglycemia and weight loss of total lignans from Fructus Arctii in KKAy mice and its mechanisms of the activity. Phytother Res 2017;10.1002/ ptr.6003.

19 Gonwa TA, Hamilton RW, Buckalew VM, Jr:: Chronic renal failure and end-stage renal disease in northwest North Carolina. Importance of analgesic-associated nephropathy. Arch Intern Med 1981;141:462-465.

-20 Kowtharapu BS, Murin R, Junemann AGM, Stachs O: Role of Corneal Stromal Cells on Epithelial Cell Function during Wound Healing. Int J Mol Sci 2018;19:E464.

-21 Liu J, Zhong Y, Liu G, Zhang X, Xiao B, Huang S, Liu H, He L: Role of Stat3 Signaling in Control of EMT of Tubular Epithelial Cells During Renal Fibrosis. Cell Physiol Biochem 2017;42:2552-2558.

22 Zhou S, Sun X, Yu L, Zhou R, Li A, Li M, Yang W: Differential expression and clinical significance of epithelialmesenchymal transition markers among different histological types of triple-negative breast cancer. J Cancer 2018;9:604-613.

23 Sengodan SK, K HS, Nadhan R, Srinivas P: Regulation of epithelial to mesenchymal transition by BRCA1 in breast cancer. Crit Rev Oncol Hematol 2018;123:74-82.

24 Brzozowa-Zasada M, Piecuch A, Michalski M, Segiet O, Kurek J, Harabin-Slowinska M, Wojnicz R: Notch and its oncogenic activity in human malignancies. Eur Surg 2017;49:199-209.

-25 Morrisey K, Evans RA, Wakefield L, Phillips AO: Translational regulation of renal proximal tubular epithelial cell transforming growth factor-beta1 generation by insulin. Am J Pathol 2001;159:1905-1915. 


\section{Cellular Physiology Cell Physiol Biochem 2018;51:2085-2097 and Biochemistry \begin{tabular}{l|l|l} 
DOl: $10.1159 / 000495827$ \\
Published
\end{tabular} 2018 The Author(s). Published by S. Karger AG, Basel

26 Murea M, Park JK, Sharma S, Kato H, Gruenwald A, Niranjan T, Si H, Thomas DB, Pullman JM, Melamed ML, Susztak K: Expression of Notch pathway proteins correlates with albuminuria, glomerulosclerosis, and renal function. Kidney Int 2010;78:514-522.

27 Walsh DW, Roxburgh SA, McGettigan P, Berthier CC, Higgins DG, Kretzler M, Cohen CD, Mezzano S, Brazil DP, Martin F: Co-regulation of Gremlin and Notch signalling in diabetic nephropathy. Biochim Biophys Acta 2008;1782:10-21.

28 Brito FC, Gosmann G, Oliveira GT: Extracts of the unripe fruit of Ilex paraguariensis as a potential chemical control against the golden apple snail Pomacea canaliculata (Gastropoda, Ampullariidae). Nat Prod Res 2018;10.1080/14786419.2018.14430841-4.

29 Li CY, Wang Q Shen S, Wei XL, Li GX: Oridonin inhibits migration, invasion, adhesion and TGF-beta1induced epithelial-mesenchymal transition of melanoma cells by inhibiting the activity of PI3K/Akt/GSK3beta signaling pathway. Oncol Lett 2018;15:1362-1372.

30 Divya T, Velavan B, Sudhandiran G: Regulation of TGF-beta/Smad Mediated Epithelial-Mesenchymal Transition by Celastrol Provides Protection against Bleomycin-induced Pulmonary Fibrosis. Basic Clin Pharmacol Toxicol 2018;10.1111/bcpt.12975

-31 Ye Y, Liu M, Yuan H, Ning S, Wang Y, Chen Z, Ji R, Guo Q Li Q, Zhou Y: COX-2 regulates Snail expression in gastric cancer via the Notch1 signaling pathway. Int J Mol Med 2017;40:512-522. 